\title{
VariaCiÓn MORFO-ANATÓMICA DE RIBES CILIATUM a LO LARGO DE Un GRADIENTE ALTITUDINAL EN EL NORTE DE LA SieRRA Nevada, MÉXICO
}

\author{
Mayte S. Jiménez-NorieGa ${ }^{1}$, Teresa Terrazas ${ }^{2}$ y Lauro López-Mata ${ }^{1,3}$ \\ ${ }^{1}$ Posgrado en Botánica, Colegio de Postgraduados, Montecillo, Estado de México, México \\ ${ }^{2}$ Instituto de Biología, Universidad Nacional Autónoma de México, México, D.F., México \\ ${ }^{3}$ Autor para la correspondencia: lauro@colpos.mx
}

\begin{abstract}
Resumen: La distribución de las especies a lo largo de gradientes altitudinales está asociada a los cambios ambientales, los cuales a su vez, inducen que las plantas modulen su morfología y anatomía. En este estudio se evaluaron los cambios morfo-anatómicos de hoja y madera de Ribes ciliatum (Grossulariaceae) en un gradiente altitudinal en el cerro Tláloc, en la región norte de la Sierra Nevada, Estado de México. Se recolectaron muestras de los 2,949 m a los 3,545 m de altitud. Se hicieron preparaciones permanentes para describir y cuantificar su anatomía; a los datos obtenidos se les aplicó análisis de varianza y de regresión múltiple. Ribes ciliatum presentó una disminución en la altura de los individuos con el incremento en altitud, asociado el estrés que provocan las bajas temperaturas, la alta intensidad lumínica y los suelos pobres. Algunos caracteres anatómicos de la hoja y madera presentaron una disminución en el sitio de mayor altitud. Sin embargo, el espesor del parénquima en empalizada, el número de estomas, la densidad de vasos y el número de radios, tuvieron un decremento gradual como se ha registrado para otras especies. Las regresiones múltiples corroboraron que la altitud fue la variable que mejor predice estas cuatro variables, junto con la densidad del dosel, la materia orgánica y la humedad del suelo. En el caso de la madera, la disminución en la densidad de vasos, junto con la presencia de vasos y anillos de crecimiento más angostos, se interpretan como ajustes a las condiciones ambientales que les permiten mantener la conducción y evitar embolismos. Además, la presencia de radios paedomórficos, en el sitio de mayor altitud, se interpreta como un estrategia para el almacenamiento. La variación morfo-anatómica encontrada en $R$. ciliatum no es lineal para todos los atributos evaluados, como se registra para otras especies fuera de los Neotrópicos.
\end{abstract}

Palabras clave: anatomía foliar, densidad de vasos, densidad estomática, Faja Volcánica Transmexicana, madera, radios paedomórficos.

\begin{abstract}
The species distribution along altitudinal gradients is associated with climate changes, promoting changes in the morphology and anatomy of the species. In this study, the morpho-anatomical changes of leaf and wood of Ribes ciliatum (Grossulariaceae) were evaluated along an altitudinal gradient in Tlaloc Mount, at the northern region of Sierra Nevada, State of Mexico. The samples were collected from 2,949 $\mathrm{m}$ up to 3,545 m elevation. Leaf and wood permanent slides were prepared to describe and quantify characters. Variance and multiple regression analyses were performed. Ribes ciliatum showed a decrease in the plant size and probably it is explained by the low temperatures, the high light intensity, and poor soils. Some leaf and wood characters showed a decrease in the site of higher elevation. However, for palisade parenchyma width, stomata density, vessel density, and number of rays, the decrement was gradual as it has been reported for other species. Multiple regressions revealed that altitude was the variable that better predicts these four variables together with canopy density, organic matter, and soil humidity. In the case of wood, the reduction on vessel density with narrower vessels and narrower width rings are considered modifications to survive under those environmental conditions allowing the plants to maintain water movement and avoid embolisms. In addition, the occurrence of paedomorphic rays at the highest elevation site is interpreted as a trait for storage. The morpho-anatomical variation found in $R$. ciliatum is not linear in most variables evaluated as it is reported for other species growing outside the Neotropics.
\end{abstract}

Keywords: foliar anatomy, paedomorphic rays, stomata density, Transmexican volcanic belt, vessel density, wood.

a distribución de las especies a lo largo de gradientes - altitudinales está asociada directamente con los cambios ambientales que ahí se presentan (Cavieres y Piper, 2004). La variabilidad ambiental se relaciona con factores tales como la radiación solar, la temperatura del aire, las propiedades del suelo y el déficit hídrico (Molina-Montenegro, 2008; Molina-Montenegro y Cavieres, 2010; Cornelius et al., 2013). En consecuencia, las plantas modulan 
sus características estructurales mediante la variación en su morfología y anatomía. Estas modificaciones suelen expresarse principalmente en las hojas y en los tallos (Carlquist, 1994; Cavieres y Piper, 2004; De Casas et al., 2007). En los tallos, parte de la variabilidad se presenta en los caracteres anatómicos de la madera, como una estrategia de supervivencia, en respuesta a condiciones estresantes (Carlquist, 1994; Fonti et al., 2010). Por otra parte, Rada et al. (1987), Molina-Montenegro y Cavieres (2010) y Zarinkamar et al. (2011), han abordado el estudio de los cambios morfo-anatómicos de la hoja en distintos gradientes ambientales del páramo tropical alpino en Santo Domingo, el matorral subandino en Chile y la región Ghean en Irán, respectivamente. Además, diversas investigaciones han mostrado cambios anatómicos en la hoja (Carlquist, 1994; Ely et al., 2005) y en la madera (van den Oever et al., 1981; Carlquist, 1994; Briceño et al,. 2000; Motomura et al., 2007; Noshiro et al., 2010), tanto a nivel de género como de especie. Estos estudios son controversiales respecto a si las estructuras morfoanatómicas son el resultado de correlaciones directamente asociadas con incrementos en la altitud. Carlquist (1994) comenta sobre la importancia de tener un mayor número de estudios que evalúen los cambios morfo-anatómicos de las especies a lo largo de gradientes altitudinales, localizados en zonas montañosas tropicales o subtropicales, ya que no se conocen las adaptaciones que pueden exisitir en estas latitudes bajo condiciones cambiantes con la altitud.

En el presente estudio se evaluaron los cambios morfo-anátomicos en la hoja y en la madera de Ribes ciliatum Humb. \& Bonpl. ex Roem \& Schult. (Grossulariaceae), entre sitios de muestreo a lo largo de una gradiente altitudinal en la región norte de la Sierra Nevada en el Estado de México. La hipótesis de trabajo fue que las estructuras morfo-anátomicas de la hoja y del tallo disminuyen sus dimensiones según se incrementa la altitud, reconociendo que tales cambios pueden ser lineales.

Ribes es un género que se reconoce como miembro de la familia Grossulariaceae, con alrededor de 150 especies de arbustos y árboles de porte bajo (Schultheis y Donaghue, 2004), de las cuales, en el Hemisferio Norte, se reconocen cerca de 100 especies. Ribes ciliatum se distribuye en los bosques de encino, de coníferas y mixtos, además de pastizales y matorrales, desde los 2,050 m hasta 3,150 m de altitud, principalmente en el centro y sur de México (Pérez-Calix, 2005). No existen descripciones de los atributos anatómicos de $R$. ciliatum, pero sí de la anatomia foliar y caulinar de otras especies del género, destacándose que la lámina foliar tiene tricomas, mesofilo bifacial con drusas y haces colaterales con una vaina parenquimatosa. La madera presenta porosidad de difusa a anular, elementos de vaso con placas de perforación escalariforme, fibrotraquiedas septadas o no septadas, y radios heterogéneos u homogéneos multiseriados (Stern et al., 1971; Pujana et al., 2008).

\section{Materiales y método}

Área de estudio. El estudio se realizó dentro de la provincia morfotectónica de la Faja Volcánica Transmexicana (Ferrusquía-Villafranca, 2007), al oriente del Estado de México, sobre el declive oeste del Cerro Tláloc, entre los $19^{\circ} 23^{\prime} 43^{\prime \prime}$ y $19^{\circ} 28^{\prime} 37^{\prime \prime}$ latitud norte, y entre los $98^{\circ} 42^{\prime}$ '51' y $98^{\circ} 48^{\prime} 12^{\prime}$ " longitud oeste (Sánchez-González y López-Mata, 2003).

Muestreo. Se seleccionaron cuatro sitios en un gradiente altitudinal de 2,994 a 3,545 m s.n.m., que contenían poblaciones de Ribes ciliatum. Es importante notar que por arriba de esta altitud no se encuentra la especie y que 2,994 m es el límite inferior de su distribución en este sitio. Se recolectaron tres individuos en cada sitio (Cuadro 1), a los que previamente se le midió in situ su altura y diámetro basal, y 15 hojas por individuo. Asimismo, se cuantificó la densidad del dosel con un densitómetro esférico (canopy-scope, Misisipi, E.U.A.) y, con base en la estructura de perennación (sensu Raunkiaer, 1934), se asignó la forma de vida. Diez hojas se colocaron extendidas en bolsas de papel y el resto se fijaron en FAA (formaldehído, alcohol etílico al 96\%, ácido acético glacial, agua; Ruzin, 1999). De cada individuo se cortó con serrote una muestra de madera en la base del tallo, a no más de $10 \mathrm{~cm}$ del nivel de suelo, y después se cortaron cilindros de 3-4 cm de longitud y se colocaron en frascos con FAA. El resto de la muestra de los tallos se etiquetó y procesó para ser depositada en la xiloteca del Instituto de Biología de la UNAM.

Laboratorio. A las diez hojas recolectadas se les midió el área foliar con un integrador de áreas (LI-3100c LI-COR, Nebraska, E.U.A.). Una vez medidas, se colocaron en una estufa a $70{ }^{\circ} \mathrm{C}$ por tres días, para después pesarse en una balanza analítica. Se calculó el área foliar específica [AFE; Garnier et al., 2001, donde AFE = área $\left(\mathrm{cm}^{2}\right)$ (peso seco $\left.(\mathrm{mg}))^{-1}\right]$.

Las muestras de hoja y madera fijadas en campo se lavaron con agua corriente. Para la hoja se realizaron cortes transversales a mano alzada y enseguida se deshidrataron con una serie de alcohol ascendente (50-100\%), se tiñeron con safranina-verde rápido (Ruzin, 1999) y montaron en resina sintética. Para hacer las observaciones de la superficie epidérmica, se separaron las epidermis de la hoja por medio

Cuadro 1. Localización geográfica de los sitios de recolecta de Ribes ciliatum a lo largo del gradiente altitudinal en el Cerro Tláloc, en el norte de la Sierra Nevada.

\begin{tabular}{cccc}
\hline Sitio & Altitud $(\mathrm{m})$ & Latitud & Longitud \\
\hline 1 & 2,945 & $19^{\circ} 26^{\prime} 55.4^{\prime \prime}$ & $98^{\circ} 46^{\prime} 39.3^{\prime \prime}$ \\
2 & 3,366 & $19^{\circ} 26^{\prime} 16.2^{\prime \prime}$ & $98^{\circ} 45^{\prime} 10.0^{\prime \prime}$ \\
3 & 3,386 & $19^{\circ} 26^{\prime} 19.0^{\prime \prime}$ & $98^{\circ} 44^{\prime} 55.9^{\prime \prime}$ \\
4 & 3,545 & $19^{\circ} 24^{\prime} 43.3^{\prime \prime}$ & $98^{\circ} 44^{\prime} 59.3^{\prime \prime}$ \\
\hline
\end{tabular}




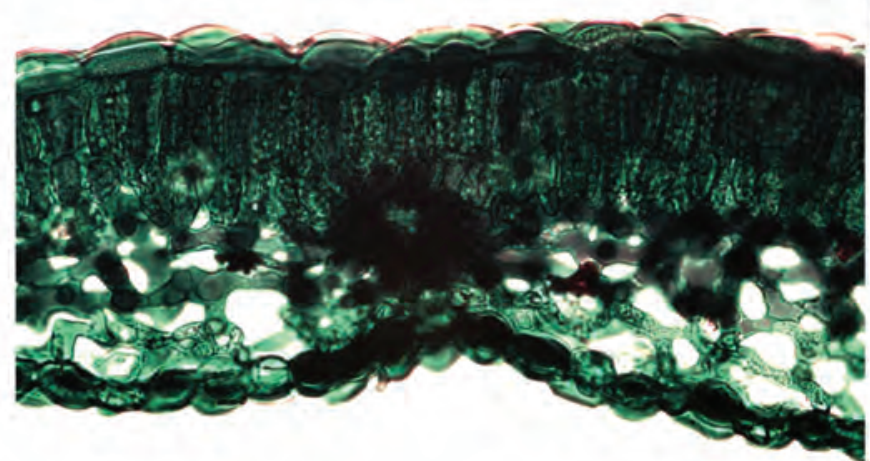

A

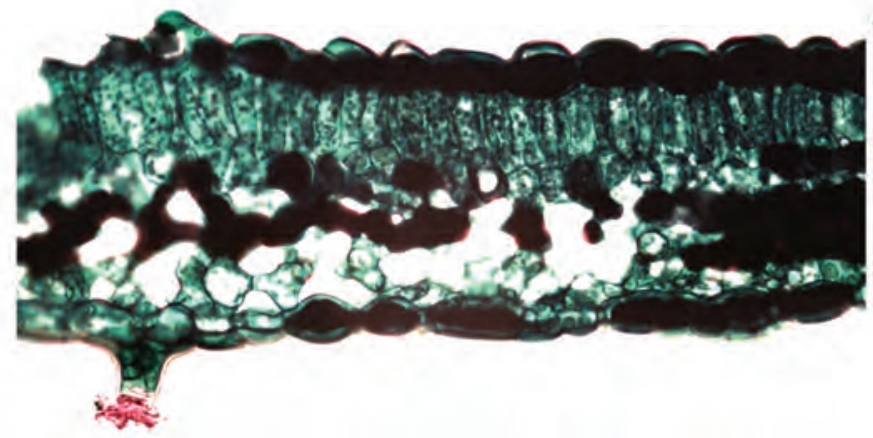

C

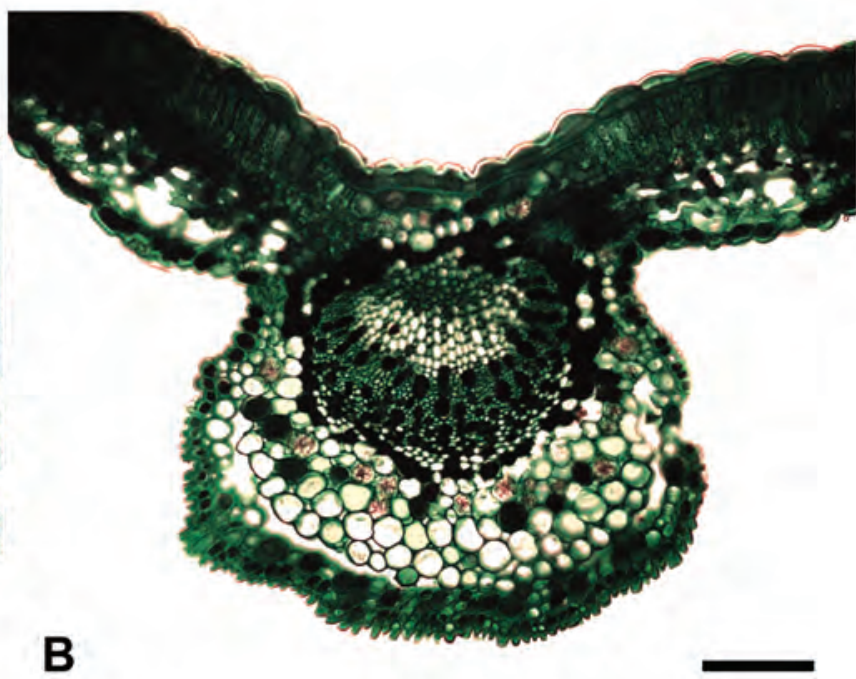

B

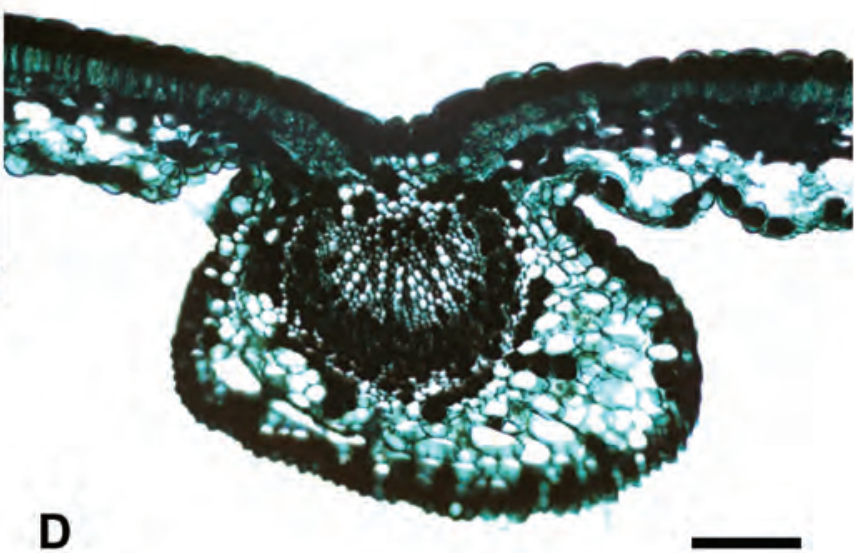

Figura 1. Anatomía de la hoja de Ribes ciliatum en el gradiente altitudinal en el cerro Tláloc, en el norte de la Sierra Nevada. A, C) Cortes transversales de la lámina. A) sitio 1 (2,949 m), C) sitio $3(3,386 \mathrm{~m})$. B, D) Vena media con el tejido vascular en un arco abierto. B) sitio $1(2,949 \mathrm{~m}), \mathrm{D})$ sitio $3(3,386 \mathrm{~m})$. Barra $=50 \mu \mathrm{m}$.

de $\mathrm{NaOH}$ al $10 \%$ y cloro comercial. Una vez que se eliminó el exceso de cloro con agua destilada, se utilizaron series ascendentes de alcoholes (50-96\%) para su deshidratación y posteriormente se tiñeron con safranina (96\%), el excedente se removió con alcohol ascendente (96-100\%) y xilol, y se montaron en resina sintética.

Para el tallo, los cilindros se dividieron en dos a través de la médula y se almacenaron en glicerina-alcohol-agua (1:1:1) hasta su ablandamiento (un mes). Posteriormente, se hicieron cortes transversales, radiales y tangenciales seriados con un micrótomo de deslizamiento (Leica 2000R, Wetzlar, Alemania) a $20 \mu \mathrm{m}$ de grosor. Todos los cortes se deshidraton en series ascendentes de alcoholes (50-96\%) y se tiñeron con safranina-verde rápido y montaron también en resina sintética. Además, se hicieron astillas, con una navaja, de la madera cercana al cámbium vascular para preparar disociados. Las astillas se colocaron en solución de
Jeffrey (Ruzin, 1999) hasta la separación de los elementos celulares, y con el material disociado se hicieron preparaciones temporales para medir la longitud de los elementos de vaso y de las fibras.

La descripción anatómica de la hoja se realizó siguiendo los términos empleados por Metcalfe y Chalk (1979), y Fahn (1982). Mientras que, para la madera se siguieron las recomendaciones de la Asociación Internacional de Anatomistas de la Madera (IAWA Committee, 1989). En los cortes transversales de la hoja se cuantificaron la anchura de la lámina, del mesofilo, del parénquima en empalizada y del parénquima esponjoso, así como alto y ancho de las células epidérmicas adaxiales y abaxiales. En ambas superficies de la epidermis se contó número de estomas, de tricomas y de células epidérmicas por $\mathrm{mm}^{2}$. Además, se calculó el índice estomático (IE), donde IE $=$ [número de estomas (número de estomas + número de células epidérmicas $\left.)^{-1}\right] \times 100(\mathrm{Sa}-$ 
MAYTe S. JimÉNEZ-NoRiega ET AL.

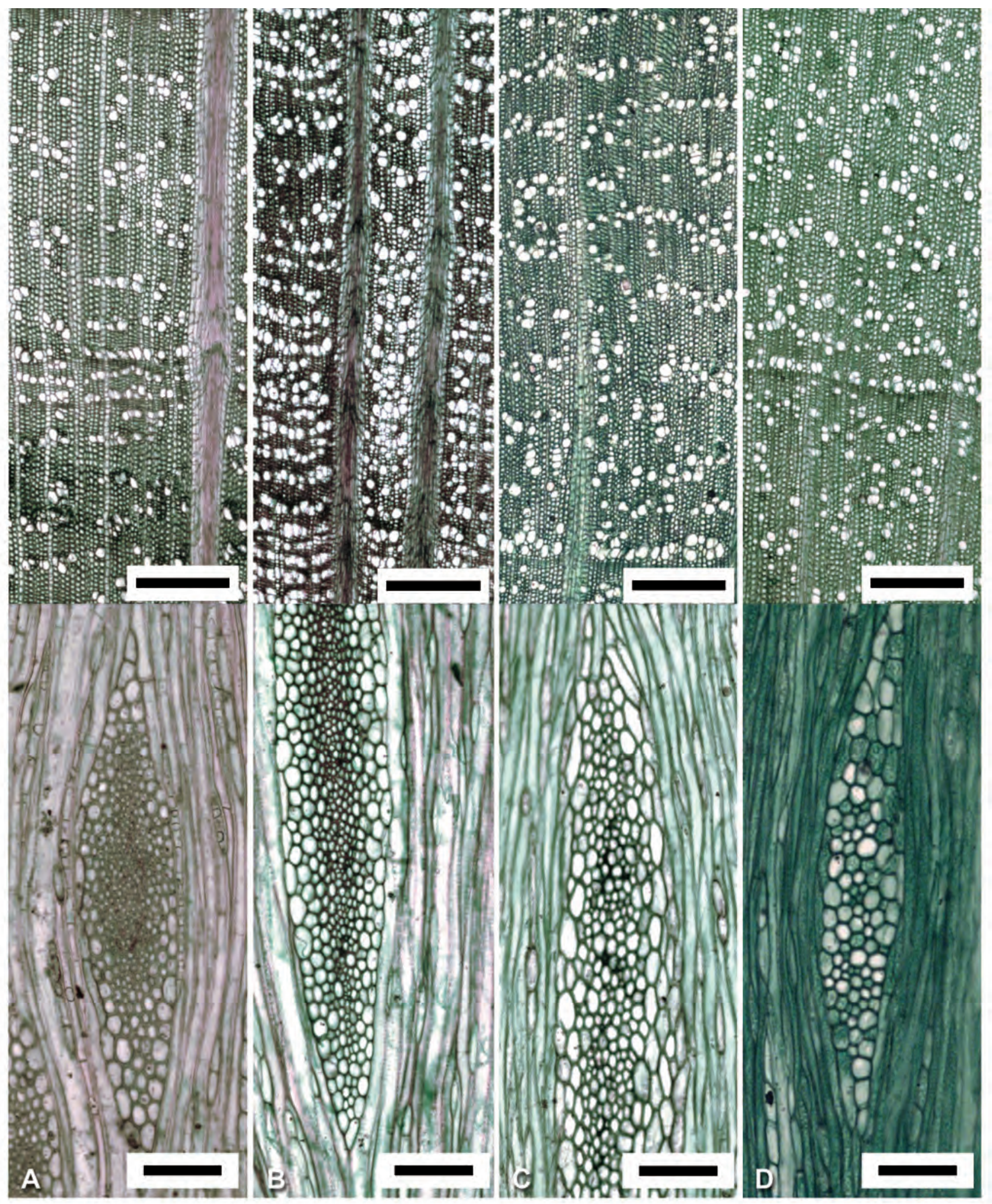

Figura 2. Madera de Ribes ciliatum en el gradiente altitudinal en el cerro Tláloc en el norte de la Sierra Nevada. A-D, superior) Porosidad anular, cortes transversales. A-D, inferior) Radios multiseriados, cortes tangenciales. A) sitio 1 (2,949 m), B) sitio 2 (3,366 m), C) sitio 3 $(3,386 \mathrm{~m}), \mathrm{D})$ sitio $4(3,545 \mathrm{~m})$. Barra $=300 \mu \mathrm{m}$ en figuras superiores; $100 \mu \mathrm{m}$ en figuras inferiores. 
Cuadro 2. Valores de la media para la altura de los individuos, el área foliar y el peso seco de la hoja de Ribes ciliatum, a lo largo del gradiente altitudinal en el cerro Tláloc, en el norte de la Sierra Nevada. Valores con letras diferentes indican diferencias significativas (Tukey, $P<0.05)$ para cada fila.

\begin{tabular}{lllll}
\hline & \multicolumn{4}{c}{ Altitud $(\mathrm{m})$} \\
& 2,949 & 3,366 & 3,386 & 3,545 \\
\hline $\begin{array}{l}\text { Altura del } \\
\text { individuo }(\mathrm{m})\end{array}$ & $2.35 \pm 0.40^{\mathrm{a}}$ & $3.2 \pm 1.16^{\mathrm{b}}$ & $3.08 \pm 0.95^{\mathrm{b}}$ & $1.46 \pm 0.35^{\mathrm{c}}$ \\
Área foliar $\left(\mathrm{cm}^{2}\right)$ & $9.29^{\mathrm{b}}$ & $11.13^{\mathrm{ab}}$ & $9.09^{\mathrm{b}}$ & $14.65^{\mathrm{a}}$ \\
Peso seco $(\mathrm{mg})$ & $7 \pm 2^{\mathrm{a}}$ & $7 \pm 2^{\mathrm{a}}$ & $6 \pm 2^{\mathrm{b}}$ & $4 \pm 3^{\mathrm{a}}$ \\
\hline
\end{tabular}

lisbury, 1928) y el índice de tricomas (IT), donde IT = [número de tricomas (número de tricomas + número de células epidérmicas $\left.)^{-1}\right] \times 100$. Para la madera se cuantificaron: densidad de vasos por $\mathrm{mm}^{2}$, diámetro tangencial y radial del lumen de vaso, longitud del elemento de vaso y de la fibra, así como número de radios por mm y, altura y anchura de radios (IAWA Committee, 1989). Tanto los caracteres foliar como de la madera se cuantificaron con ayuda de un analizador de imágenes (Image-Pro Plus versión 3.1, Media Cybernetics, Rockville, E.U.A.).

Análisis estadísticos. A los datos se les aplicó prueba de normalidad y de homogeneidad de varianzas. Se practicaron, para cada uno de los caracteres morfológicos y anatómicos de la hoja y de la madera de Ribes ciliatum, análisis de varianza del modelo general linear seguido del análisis de comparación de medias de Tukey. Además, a los caracteres que mostraron una tendencia con respecto al gradiente altitudinal se les aplicó un análisis de regresión múltiple por pasos, con el propósito de detectar si tales tendencias se encontraban relacionadas con la altitud, la densidad del dosel, la materia orgánica y el punto de marchitez permanente (humedad) del suelo, tanto del horizonte A como del B. De acuerdo a Sánchez-González y López-Mata (2003), tanto la materia orgánica como el punto de marchitez permanente del suelo tienen efectos en la distribución de las especies en el mismo gradiente altitudinal estudiado. Los datos originales de ambas variables fueron generados y tomados del trabajo de Sánchez-González y López-Mata (2003). Todos los análisis estadísticos fueron llevados a cabo con el paquete estadístico SAS ver. 9.2 (SAS, 2008).

\section{Resultados}

Características morfólogicas y variación a lo largo del gradiente altitudinal. Ribes ciliatum es una especie fanerófita caducifolia, con yema de renuevo por arriba de los $25 \mathrm{~cm}$, tiene hojas alternas, pecioladas, lobadas, margen irregularmente dentado, pubescente en ambas superficies y venación actinódroma marginal muy evidente en el envés. La altura de los individuos fue muy variable (Cuadro 2), presentando sus valores más pequeños en los extremos del gradiente y valores máximos a altitudes intermedias. Por otra parte, el área foliar sólo mostró un incremento desproporcionado en el extremo superior del gradiente; mientras que el peso seco presentó una disminución hacia los sitios de mayor altitud (Cuadro 2).

Anatomía foliar. Cutícula lisa y delgada, epidermis adaxial y abaxial simple con contenidos oscuros (Figura 1), tricomas simples, estomas en ambas superficies de la hoja, el resto de las células epidérmicas rectangulares y más cortas en la superficie abaxial (Cuadro 3). Mesofilo bifacial, parénquima en empalizada de uno (sitio 3) o dos (sitio 1, 2, 4) estratos celulares, parénquima esponjoso cerrado o laxo, con abundantes contenidos oscuros y drusas. Haces vasculares colaterales con una vaina parenquimatosa con contenidos oscuros. Vena media con cutícula lisa y delgada; epidermis simple, epidermis abaxial pronunciadamente cóncava con tricomas simples, células de forma rectangular, epidermis adaxial con células cuadrangulares, con pared periclinal externa colenquimatosa; tejido vascular en un haz de arco abierto, rodeado por parénquima con contenidos oscuros y drusas, el xilema con elementos traqueales arreglados en hileras radiales, el floema con elementos del tubo criboso y células acompañantes que se encuentran hacia la superficie abaxial, separadas por parénquima con contenidos también oscuros.

Anatomía de la madera. Anillos de crecimiento delimitados por vasos de diámetro amplio en la madera temprana y, en la madera tardía por fibras con lúmenes radiales reducidos (Figura 2). Anillos de crecimiento anchos (sitios 1 y 3) o angostos (sitios 2 y 4). Porosidad anular. Madera temprana con vasos agrupados (residuos 1 y 3 ) o casi solitarios (sitios 2 y 4), de forma ligeramente ovalada, diámetro tangencial

Cuadro 3. Valores de la media para el tamaño de la epidermis abaxial (aba) y adaxial (ada) de la hoja de Ribes ciliatum a lo largo del gradiente altitudinal en el cerro Tláloc, en el norte de la Sierra Nevada. Valores con letras diferentes muestra diferencia significativas (Tukey, $P<0.05)$.

\begin{tabular}{|c|c|c|c|c|}
\hline \multirow[t]{2}{*}{ Carácter anatómico } & \multicolumn{4}{|c|}{ Altitud (m s.n.m.) } \\
\hline & 2,949 & 3,366 & 3,386 & 3,545 \\
\hline Ancho adaxial & $33.52 \pm 6.74^{\mathrm{a}}$ & $29.63 \pm 6.47^{\mathrm{ab}}$ & $26.14 \pm 6.87^{\mathrm{b}}$ & $32.02 \pm 10.76^{a}$ \\
\hline Alto adaxial & $22.30 \pm 3.10^{\mathrm{a}}$ & $21.41 \pm 3.78^{a}$ & $17.61 \pm 2.84^{\mathrm{b}}$ & $18.53 \pm 18.53^{b}$ \\
\hline Ancho abaxial & $23.50 \pm 6.60^{\mathrm{ab}}$ & $22.66 \pm 6.70^{\mathrm{ab}}$ & $21.09 \pm 5.41^{\mathrm{ab}}$ & $25.85 \pm 5.23^{\mathrm{a}}$ \\
\hline Alto abaxial & $18.12 \pm 2.73^{a}$ & $15.31 \pm 2.66^{b}$ & $15.60 \pm 3.23^{\mathrm{b}}$ & $18.60 \pm 3.29^{a}$ \\
\hline
\end{tabular}



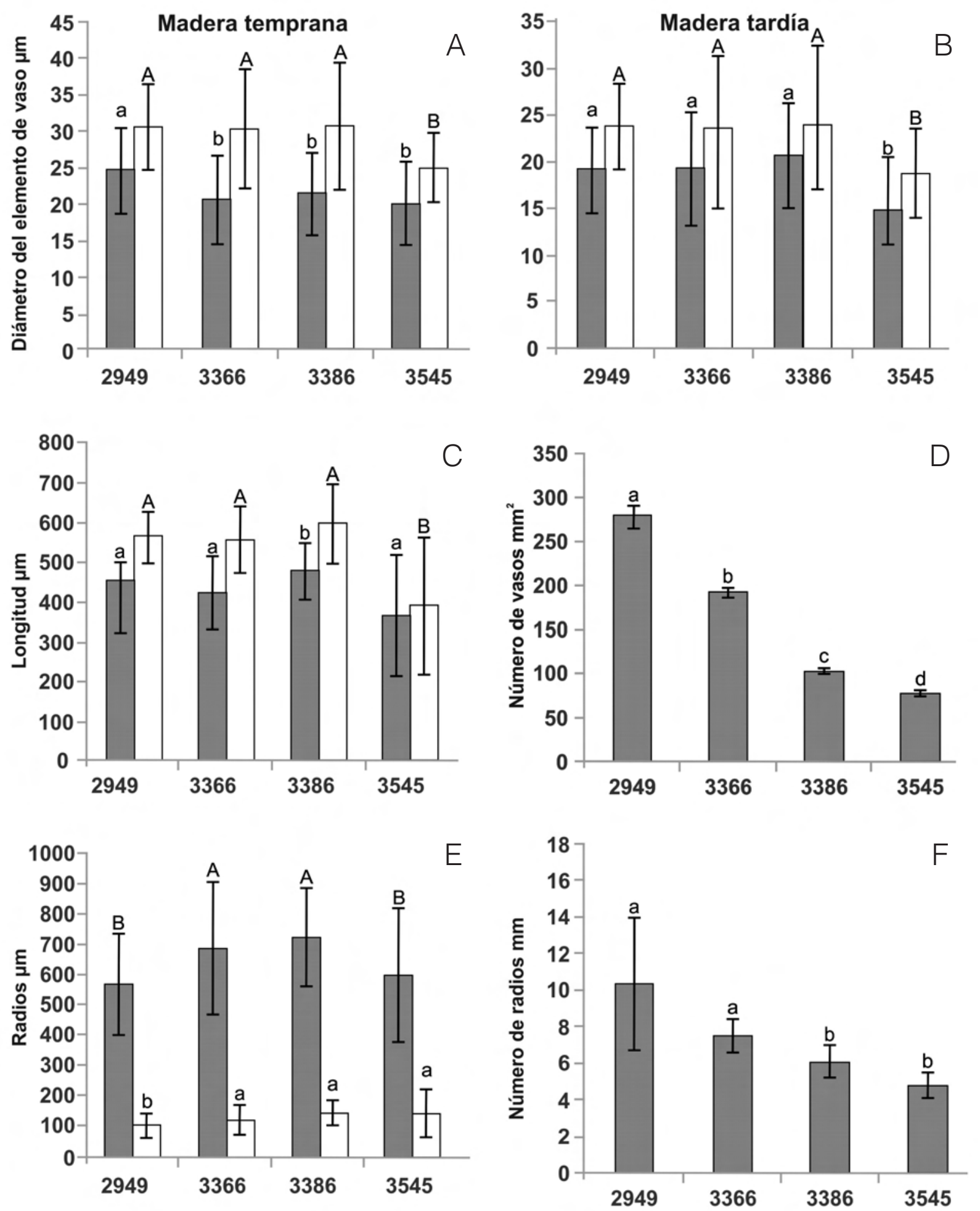

Figura 3. Valores de la media y diferencias significativas para los caracteres anatómicos de la madera de Ribes ciliatum en el gradiente altitudinal en el cerro Tláloc, en el norte de la Sierra Nevada. A) Diámetro radial (blanco) y tangencial (gris) de los vasos de la madera temprana. B) Diámetro radial (blanco) y tangencial (gris) de los vasos de la madera tardía. C) Longitud del elemento de vaso (gris) y de fibras (blanco). D) Densidad de vasos. E) Altura (gris) y anchura (blanco) de radios. F) Número de radios. Letras diferentes (minúsculas o mayúsculas) indican diferencias significativas (Tukey, $P<0.05$ ). 

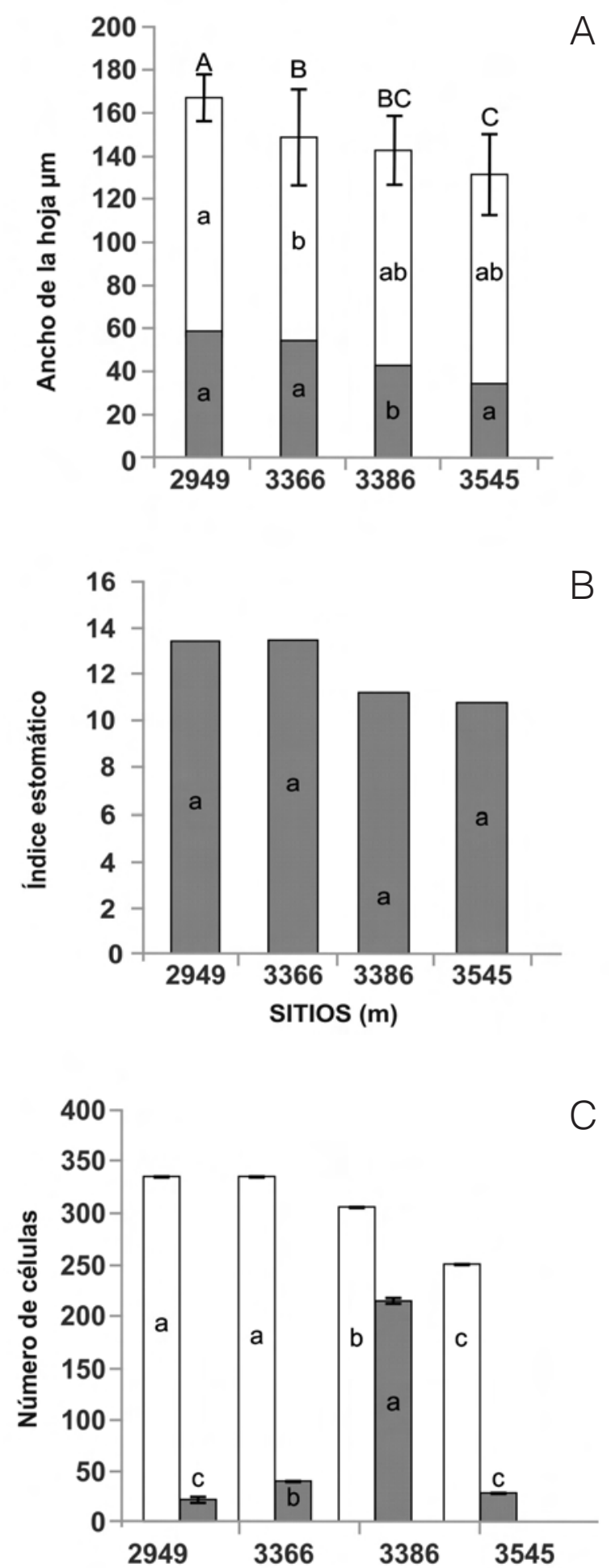

Figura 4. Valores de la media y diferencias significativas para tres caracteres anatómicos de la hoja de Ribes ciliatum en el gradiente altitudinal en el cerro Tláloc, en el norte de la Sierra Nevada. A) Ancho de la lámina: parénquima en empalizada (gris) y parénquima esponjoso (blanco). B) Índice estomático. C) Densidad de tricomas (gris) y número de estomas, por $\mathrm{mm}^{2}$ (blanco). Letras diferentes (minúsculas o mayúsculas) indican diferencias significativas (Tukey, $P<0.05$ ). que varía entre sitios, de 19.9-24.44 $\mu \mathrm{m}$, y diámetro radial de 24.80-30.36 $\mu \mathrm{m}$ (Figura 3). Madera tardía: con 78-279 vasos $\mathrm{mm}^{-2}$ entre sitios, vasos de forma ovalada o circular, diámetro tangencial entre sitios de 14.89-20.65 $\mu \mathrm{m}$ y diámetro radial de 18.73-23.82 $\mu \mathrm{m}$. Elementos de vasos con placas de perforación escalariformes y simples, punteaduras intervasculares alternas y de $368-479 \mu \mathrm{m}$ en longitud. Fibrotraqueidas de 393-598 $\mu \mathrm{m}$ de longitud. Radios 6-12 por mm, en su mayoría heterogéneos multiseriados, de 5-17 series; de 569-723 $\mu \mathrm{m}$ en altura y 103-144 $\mu \mathrm{m}$ de anchura, con pocas células erectas en los extremos y el resto procumbentes (sitios 1-3) o únicamente erectas (sitio 4).

Variación anátomica a lo largo del gradiente altitudinal. El espesor de la hoja mostró un decremento significativo en la proporción del parénquima en empalizada y del parénquima esponjoso $(P<0.0001)$ conforme aumenta la altitud (Figura $4)$; mientras que, el ancho y alto de las células epidérmicas de la superficie adaxial y abaxial no exhibió ningún patrón de variación con la altitud, pero sí hubo diferencias significativas entre algunos sitios (Cuadro 3). El índice estomático disminuyó cuando incrementó la altitud; sin embargo, no presentó diferencias significativas $(P>0.05)$ entre los sitios. El número de estomas presentó también disminuciones conforme se incrementó la altitud, pero con diferencias altamente significativas $(P<0.0001)$ entre los sitios; en cambio, el número de tricomas mostró un incremento abrupto en el sitio 3 a 3,386 m de altitud (Figura 4).

La madera temprana y tardía presentó un decremento en el diámetro radial y tangencial de los vasos en el sitio de mayor altitud (3,545 m); así como para la longitud de los elemento de vaso y de las fibras. Sólo para la densidad de vasos y número de radios se presentó una disminución gradual conforme aumenta la altitud (Figura 3). La altura de los radios presentó una disminución hacia los extremos del gradiente altitudinal y el ancho de los radios disminuyó en el sitio de menor altitud $(2,994 \mathrm{~m})$ del gradiente altitudinal (Figura 3).

Los análisis de regresión multiple revelaron que la altitud fue la variable más importante que explica el más alto porcentaje de la variabilidad, tanto en el espesor del parénquima en empalizada, como en el número de estomas y el número de tricomas (Cuadro 4). Por otra parte, la variación en la densidad de vasos y el número de radios de la madera también se explica en función de la altitud y, en menor medida, con la incorporación al modelo de la densidad del dosel, de la materia orgánica y de la humedad del suelo.

\section{Discusión}

La disminución del tamaño de los individuos que se observó en Ribes ciliatum (fanérofita), con el incremento de la altitud, se reconoce como una de las estrategias más comúmente observada en las especies para tolerar y resistir el estrés 
Cuadro 4. Regresión múltiple por pasos de las variables altitud, dd: densidad del dosel, $\mathrm{Mo}_{1}$ : materia orgánica, $\mathrm{Pmp}_{2}$ : punto de marchitez permanente. Los subíndices, $y_{2}$ indican los horizonte del suelo $A_{1} y$ $A_{2}$, respectivamente, sobre las variables de la hoja y de la madera de Ribes ciliatum. Únicamente se presentan las variables significativas seleccionadas por el modelo.

\begin{tabular}{lllll}
\hline Carácter anatómico & Variables & $\begin{array}{l}\mathrm{R}^{2} \text { del } \\
\text { modelo }\end{array}$ & $\mathrm{R}^{2}$-parcial & $P$ \\
\hline Ancho parénquima & altitud & 0.25 & 0.25 & 0.0001 \\
en empalizada & $\mathrm{Mo}_{1}$ & 0.30 & 0.05 & 0.0036 \\
& $\mathrm{dd}$ & 0.38 & 0.07 & 0.0003 \\
Densidad de estomas & altitud & 0.15 & 0.15 & 0.0020 \\
& dd & 0.31 & 0.15 & 0.0008 \\
& $\mathrm{Mo}_{1}$ & 0.36 & 0.05 & 0.0380 \\
Número de tricomas & altitud & 0.81 & 0.81 & 0.0001 \\
& $\mathrm{Mo}_{1}$ & 0.83 & 0.03 & 0.0040 \\
& altitud & 0.37 & 0.37 & 0.0001 \\
Número de radios & $\mathrm{Mo}_{1}$ & 0.44 & 0.07 & 0.0001 \\
& & & & \\
Densidad de vasos & altitud & 0.50 & 0.50 & 0.0001 \\
& dd & 0.54 & 0.04 & 0.0001 \\
& $\mathrm{PMP}_{2}$ & 0.57 & 0.03 & 0.0001 \\
\hline
\end{tabular}

(Cavieres y Piper, 2004). La tendencia de los individuos a disminuir en tamaño conforme aumenta la altitud, también ha sido reportada por Briceño et al. (2000) para dos especies del género Lupinus en regiones intertropicales.

A pesar de que el área foliar y el peso seco exhibieron diferencias significativas entre los sitios donde se distribuye Ribes ciliatum, no mostraron ningún patrón de variación. Esto contrasta con lo reportado para Phacelia secunda (Molina-Montenegro y Cavieres, 2010), especie que presentó mayor área foliar a menor altitud, considerándolo una estrategia morfológica para la resistencia al estrés de las estructuras internas.

El índice estomático disminuyó en los sitios de mayor altitud, sin que haya diferencias significativas entre los sitios. Los resultados de Ribes ciliatum difieren de lo reportado para Lupinus merideanus, donde el índice estomático es más bajo a los 3,000 m de altitud (Briceño et al., 2000). El número de tricomas exhibió una variabilidad bimodal con incrementos en la altitud. Esta variación difiere sustancialmente de lo registrado para otras especies, en las cuales el número de tricomas se incrementa a mayores altitudes $(\mathrm{Ca}-$ vieres, 2000; Molina-Montenegro, 2008; Molina-Montenegro y Cavieres, 2010). Se ha documentado que los tricomas funcionan como barreras protectoras a la radiación solar, debido a que hay un aumento en la exposición de las hojas a la radiación solar a medida que se asciende altitudinalmente. La disminución en el número de tricomas hacia los extremos del gradiente se puede deber a que a menor altitud hay una baja incidencia de la luz ultra violeta; mientras que a mayor altitud, el aumento en el contenido de pigmentos (Figura 1) sustituye la función de los tricomas (Molina-Montenegro, 2008). Además, se observó un incremento desproporciona- do en la densidad de tricomas en el sitio 3 (3,386 m), lo que sugiere una mayor exposición de los individuos de $R$. ciliatum a la luz y a las bajas temperaturas (Körner, 2003). Körner et al. (1986) y Chapolagh et al. ( 2013) reportaron correlaciones positivas entre la densidad estomática y la altitud, lo que contrasta con lo encontrado para $R$. ciliatum, que tiene un escalamiento negativo con la altitud. El decremento en la densidad estomática seguramente está asociado a la disminución del parénquima en empalizada y esponjoso de $R$. ciliatum a mayor altitud. La reducción en la lámina foliar que se registró en $R$. ciliatum, al incrementar la altitud, se puede interpretar como las modificaciones asociadas a la reducción en la talla de los individuos (Briceño et al., 2000). Además, el parénquima esponjoso fue más laxo en los sitios 1 y 2, lo que se asocia a microambientes mesomórficos (Ely et al., 2005). El parénquima esponjoso más laxo permite que en un mayor número de espacios intercelulares se acumule aire y este pueda ser empleado por la planta como amortiguador a cambios bruscos de temperatura; sin embargo, se requieren estudios ecofisiológicos que confirmen esta interpretación y que permitan entender, también, la asociación entre el cambio en la densidad estomática y el espesor del parénquima en empalizada, que seguramente favorecen ajustes de la fotosíntesis en cada sitio.

Los caracteres anátomicos de la madera: densidad de vasos y número de radios, resultaron ser los más sensibles al gradiente altitudinal, decreciendo ambos conforme aumenta la altitud. Tanto la densidad del dosel (más abierto a mayor altitud), como la reducción de la materia orgánica y la disponibilidad de agua en el suelo, según se incrementa la altitud, contribuyeron a dicho decremento (Cuadro 4). La disminución en la densidad de los vasos se ha reportado también para Lobelia gibberoa como indicador de xeromorfia, dado que esta especie se distribuye en una zona alpina (Carlquist, 1994). También para el diamétro de los vasos de Ribes ciliatum se registró una disminución con el incremento en la altitud, tanto en los vasos de la madera temprana como tardía. La reducción en el diámetro de los vasos conforme aumenta la altitud se ha reportado en otras especies (van den Oever et al., 1981; Lens et al., 2004; Motomura et al., 2007; Noshiro et al., 2010). Estos autores atribuyen la reducción del diámetro y su incremento en número a los factores estresantes como la escasez de agua en el suelo y su compensación para evitar embolismos. Sin embargo, el decremento en diámetro de los vasos en $R$. ciliatum no fue gradual conforme aumentaba la altitud, ya que en el sitio 3 , a 3,386 m de altitud, se presentó un mayor diámetro de vasos. Este es el único sitio donde un mayor diámetro se relaciona con un menor número de vasos, como se ha registrado en la literatura (Carlquist, 2001). Cabe señalar que el ancho de los anillos de crecimiento se reduce en los sitios 2 y 4 , lo que sugiere que las condiciones de crecimiento en estos sitios son menos favorables para la especie y que la actividad de cámbium vascular en ambos sitios es más corta. 
La reducción de la longitud de las fibras con el incremento en altitud no fue gradual, como lo mencionado por van den Oever et al. (1981) para especies del género Symplocos, y Motomura et al. (2007) para Ephedra pachyclada, pero seguramente está relacionado con la disminución en la talla de los individuos como en Lupinus eromonomos (Briceño et al., 2000). Con respecto a los radios, tienden a ser menos altos hacia los extremos del gradiente altitudinal, lo que contrasta con las correlaciones positivas para la altura de los radios en otras especies a mayor altitud (Arias y Terrazas, 2001; Moya y Tomazello, 2007). Distintivamente, la madera de Ribes ciliatum presenta radios paedomorficos a mayor altitud. Este tipo de radios es semejante al que Carlquist (1994) observó en especies de Espeletia y Lobelia, a elevadas altitudes y cuya presencia está asociada con una mayor capacidad de almacenamiento de reservas (Carlquist, 1994). Además, los radios paedomórficos posiblemente son un trueque, considerando que su número se reduce a mayor altitud.

\section{Conclusiones}

El tamaño de los individuos y cuatro características anatómicas, tanto de la hoja como de la madera, presentaron un escalamiento negativo gradual con la altitud, tal como se ha reportado para otras especies fuera de los Neotrópicos; además, la densidad del dosel, la materia orgánica y la humedad del suelo contribuyen a la explicación de estas variables. Otros rasgos de morfo-anatómicos también disminuyen sus dimensiones, pero lo hacen hacia ambos extremos del gradiente altitudinal estudiado; por lo que su análisis en otras especies, en el mismo gradiente altitudinal del cerro Tláloc, permitirá confirmar si tal variabilidad en los caracteres morfo-anatómicos tiende a presentar un patrón similar.

\section{Agradecimientos}

El primer autor agradece el apoyo al Consejo Nacional para la Ciencia y Tecnologia por la beca otorgada. Al posgrado de Bótanica del Colegio de Postgraduados y al Laboratorio de Botánica Estructural del Instituto de Biología por su apoyo para la realización de este trabajo. A Julio César Montero Rojas y Diana Ramírez por el diseño gráfico. A los dos revisores anónimos que hicieron criticas y sugerencias que permitieron mejorar substancialmente el manuscrito final.

\section{Literatura citada}

Arias S. y Terrazas T. 2001. Variación en la anatomía de la madera de Pachycereus pecten-aborigenum (Cactaceae). Anales del Instituto de Biología, Universidad Nacional Autónoma de México, Serie Botánica 72:157-169.

Briceño B., Azócar A., Fariñas M. y Rada F. 2000. Características anatómicas de dos especies de Lupinus L. de los Andes venezolanos. Pittieria 29,30:21-35.

Carlquist S. 1994. Anatomy of tropical alpine plants. En: Rundel
P.W., Smith A.P. y Meinzer F.C. Eds. Tropical Alpine Enviroments: Plant Form and Function, pp. 111-125, Cambridge University Press, Cambridge.

Carlquist S. 2001. Comparative Wood Anatomy: Systematic, Ecological, and Evolutionary Aspects of Dicotyledon Wood. $2^{\mathrm{a}} \mathrm{ed}$. Springer-Verlag, Berlín.

Cavieres L.A. 2000. Variación morfológica de Phacelia secunda J.F. Gmel. (Hydrophyllaceae) a lo largo de un gradiente altitudinal en Chile central. Gayana Botanica 57:89-96.

Cavieres L.A. y Piper F.I. 2004. Determinantes ecofiosiológicos del límite altitudinal de los árboles. En: Cabrera H.M. Ed. Fisiología Ecológica en Plantas: Mecanismos y Respuestas a Estrés en los Ecosistemas, pp. 221-234, Ediciones de la Universidad Católica de Valparaíso, Valparaíso.

Chapolagh P.F., Gholamali J.S., Sonboli A., Zarafshar M. y Bruschi P. 2013. Leaf macro- and micro-morphological altitudinal variability of Carpinus betulus in the Hyrcanian forest (Iran). Journal of Forestry Research 24:301-307.

Cornelius C., Estrella N., Franz H. y Menzel A. 2013. Linking altitudinal gradients and tempeture responses of plant phenology in the Bavarian Alps. Plant Biology 15(Supl.):57-69.

De Casas R.R., Vargas P., Pérez-Corona E., Manrique E., Quintana J.R., García-Verdugo C. y Balaguer L. 2007. Field patterns of leaf plasticity in adults of the long-lived evergreen Quercus coccifera. Annals of Botany 100:325-334.

Ely F., Torres F. y Gavira J. 2005. Morfología y anatomía foliar de Monochaetum meridense (Melastomataceae). Acta Botánica Venezuelica 28:197-212.

Fahn A. 1982. Anatomía Vegetal. Pirámide, Madrid.

Ferrusquía-Villafranca I. 2007. Ensayo sobre la caracterización y significación biológica. En: Luna V.I., Morrone J.J. y Espinosa D. Eds. Biodiversidad de la Faja Volcánica Transmexicana, pp. 7-23, Universidad Nacional Autónoma de México, México, D.F.

Fonti P., von Arx G., García-González I., Eilmann B., Sass-Klaassen U., Gärtner H. y Eckstein D. 2010. Studying globlal change through investigation of the plastic responses of xylem anatomy in tree rings. New Phytologist 185:42-53.

Garnier E., Shipley B., Roumet C. y Laurent G. 2001. A standardized protocol for the determination of specific leaf area and leaf dry matter content. Funtional Ecology 15:688-695.

IAWA Committee. 1989. IAWA list of microscopic features for hardwood identification: with an appendix on non-anatomical information. IAWA Bulletin New Series 10:219-332.

Körner C. 2003. Plant Alpine Life: Functional Plant Ecology of High Mountain Ecosystems. Springer-Verlag, Berlín.

Körner C., Bannister P. y Mark A.F. 1986. Altitudinal variation in stomatal conductance, nitrogen content and leaf anatomy in different plant life forms in New Zealand. Oecologia 69:577-588.

Lens F., Luteyn J.L., Smets E. y Jansen S. 2004. Ecological trends in the wood anatomy of Vaccinioideae (Ericaceae s. 1.). Flora 199:309-319.

Metcalfe C.R. y Chalk L.1979. Anatomy of the Dicotyledons: Systematic Anatomy of Leaf and Stem. Vol I. $2^{\text {a }}$ ed. Clarendon Press, Oxford.

Moya R.R. y Tomazello F.M. 2007. Relationship between anatomical features and intra-ring wood density profiles in Gmelina arborea applying X-ray densitometry. Cerne 13:384-392.

Molina-Montenegro M.A. 2008. Variación de las pubescencia foliar en plantas y sus implicaciones funcionales a lo largo de gradientes altitudinales. Ecosistemas 17:146-154. 


\section{MAYTe S. JimÉNEZ-NoRIEGA ET AL.}

Molina-Montenegro M.A. y Cavieres L.A. 2010. Variación altitudinal de los atributos morfo-fisiológicos en dos especies de plantas alto-andinas y sus implicancias contra la fotoinhibición. Gayana Botánica 67:1-11.

Motomura H., Noshiro S. y Mikage M. 2007. Variable wood formation and adaptation to the alpine enviroment of Ephedra pachyclada (Gnetales: Ephedraceae) in the Mustang District, Western Nepal. Annals of Botany 100:315-324.

Noshiro S., Ikeda H. y Joshi L. 2010. Distinct altitudinal trends in the wood structure of Rhododendron arboreum (Ericaceae) in Nepal. IAWA Journal 31:443-456.

Pérez-Calix E. 2005. Grossulariaceae. Flora del Bajío y de Regiones Adyacentes 138:1-15.

Pujana R.R., Burrieza H.P. y Castro M.A. 2008. Wood anatomy of Ribes magellanicum (Grossulariaceae). Boletín Sociedad Argentina de Botánica 43:61-65.

Rada F., Goldstein G., Azocar A. y Torres F. 1987. Supercooling along an altitudinal gradient in Espeletia schultzii, a caulescent giant rosette species. Journal of Experimental Botany 38:491-497.

Raunkiaer C. 1934. The Life Forms of Plants and Statistical Plant Geography. Clarendon Press, Oxford.

Ruzin S.E. 1999. Plant Microtechnique and Microscopy. Oxford University Press, Oxford.

Editora asociada: Silvia Aguilar Rodríguez

Recibido: 2 de abril de 2014

Aceptado: 25 de agosto de 2014
Salisbury E.J. 1928. On the causes and ecological significance of stomatal frecuency, with special reference to woodland flora. Philosophical Transactions of the Royal Society of London, Series $B$ 216:1-65.

Sánchez-González A. y López-Mata L. 2003. Clasificación y ordenación de la vegetación del norte de la Sierra Nevada, a lo largo de un gradiente altitudinal. Anales del Instituto de Biología, Universidad Nacional Autónoma de México, Serie Botánica 74:47-71.

SAS. Statistical Analysis System. 2008. SAS ver. 9.2. Statistical Analysis System, Cary.

Schultheis L.M. y Donaghue M.J. 2004. Molecular phylogeny and biogeography of Ribes (Grossulariaceae), with an emphasis on gooseberries (subg. Grossularia). Systematic Botany 29:77-96.

Stern W.L., Sweitzer E.M. y Phipps R.E. 1971. Comparative anatomy and systematics of woody Saxifragaceae. Ribes. Journal of the Linnean Society Botany 63:215-237.

van den Oever L., Baas P. y Zandee M. 1981. Comparative wood anatomy of Symplocos and latitude and altitude of provenance. IAWA Bulletin New Series 2:3-24.

Zarinkamar F., Tajik S. y Soleimanpour S. 2011. Effects of altitude on anatomy and concentration of crocin, pricrocrocin and safranal in Crocus sativus L. Australian Journal of Crop Science 5:831-838. 